\title{
Casio Longino, fr. 42.75 P.-B. ¿Un gramático llamado Odiseo o un error textual?: una propuesta de corrección*
}

\author{
Javier Verdejo Manchado \\ Universidad de Oviedo \\ verdejojavier@uniovi.es
}

\section{Cassius Longinus, fr. 42.75 P.-B. A grammarian called Odysseus or a textual error?: a proposal for correction}

El gramático del s. III Casio Longino en su introduc-

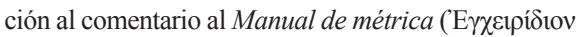

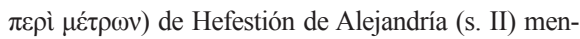

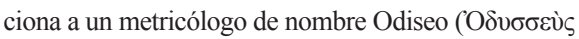
ó $\mu \varepsilon \tau$ рıкós), que no es conocido por ninguna otra fuente. Este artículo pretende identificar a este autor y dar una explicación satisfactoria a su mención en la obra de Longino. Ahora bien, la peculiaridad del nombre, que no está testimoniado como antropónimo fuera de la épica, así como su aparición en Longino, quien en su comentario sólo cita a autores teóricos que utilizó como fuente directa, nos han

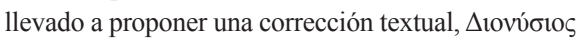

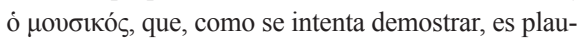
sible paleográficamente y coherente desde el punto de vista de la identificación.

Palabras clave: Longino; metricología; Hefestión; Odiseo; Dionisio.
The 3th century grammarian Cassius Longinus in his introduction to the commentary to He-

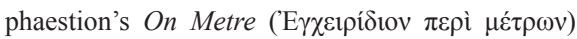
mentions a writer on meter named Odysseus

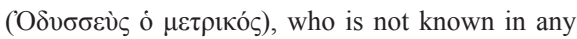
other source. This paper aims to identify this author and give a satisfactory explanation to his mention by Longinus. However, the peculiarity of the name, which is not attested as anthroponym out of the epic genre, and its notice in Longinus, who in his commentary only quotes technical authors used as direct sources, have led us to propose a textual

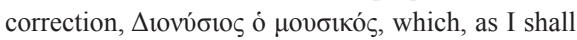
try to prove, is paleographically plausible and consistent from the point of view of the identification.

Key words: Longinus; metrics; Hephaestion; Odysseus; Dionysius.

* Este artículo ha sido elaborado en el marco del proyecto de investigación «La tradición literaria griega en los ss. III-IV d.C. gramáticos, rétores y sofistas como fuentes de la literatura greco-latina (II)», financiado por el MINECO Ref. FFI2014-52808-C2-1-P. Una primera versión del mismo fue presentada como comunicación en las XXIII Jornadas de Filología Clásica, Oviedo 2014. El autor quiere dar las gracias a la Dra. L. Rodríguez-Noriega Guillén y al Dr. Miguel Bobo de la Peña por su útiles consejos y sugerencias, que, sin duda, han contribuido a mejorar este artículo. 
Las vicisitudes y predilecciones de la tradición manuscrita han querido que la amplia producción literaria del polígrafo y polifacético gramático del s. III d.C. Casio Longino haya quedado reducida a una colección de obras incompletas, atribuciones dudosas y fragmentos de trasmisión indirecta, que en el mejor de los casos apenas alcanzan el centenar de páginas en una edición moderna. Resulta, además, que las fuentes que transmiten estos retazos suelen ser únicas, por lo que su lectura pocas veces puede ser cuestionada más allá de pequeños errores paleográficos. De este modo, en el caso que nos ocupa, la mención por parte de Longino de un autor tan enigmático y desconocido como es el metricólogo Odiseo generalmente ha sido acogida sin apenas crítica y consideración.

El nombre de Odiseo es mencionado por Longino a propósito de una

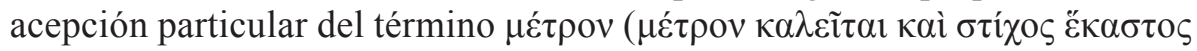

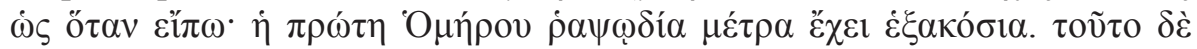

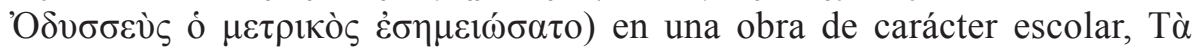

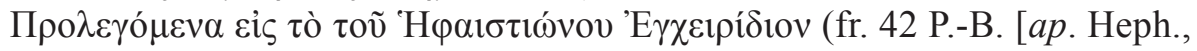
pp. 81-89 Consbr.]). Se trata de un comentario al Manual de métrica

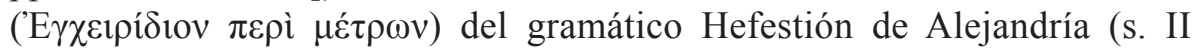
d.C.), que ha sido transmitido de manera parcial — la introducción y el inicio del comentario - únicamente por algunos manuscritos de la propia obra de Hefestión ${ }^{1}$, también junto con otros comentarios escolásticos de época bizantina.

Por otro lado, en el Anonymus Ambrosianus de re metrica (p. 231,4-29 Studemund), un breve compendio bizantino del s. XIII que contiene algunas precisiones métricas sacadas de otros autores ${ }^{2}$, encontramos una paráfrasis de

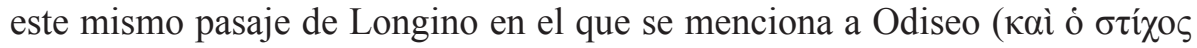

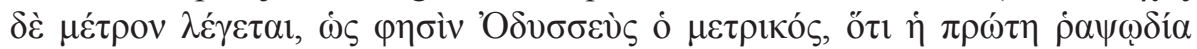

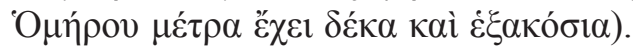

La lectura de este nombre en los manuscritos de la obra de Longino es unánime y no plantea problemas, de modo que generalmente ha sido aceptada de facto por los distintos editores modernos ${ }^{3}$. En lo que se refiere al

${ }^{1}$ Sobre la transmisión de la obra de Longino, v. infra, y n. 23.

2 Para más detalles sobre este tratado, v. infra, y n. 24.

3 Sic Vaucher 1854, p. 298, Westphal 1866, p. 87, Consbruch 1906, p 84, Patillon y Brisson 2001, p. 182, Brisson y Patillon 1998, p. 3031, Männlein-Robert 2001, pp. 567 y 569. Tan sólo Gaisford $1855^{2}$, p. 146, n. c, recoge —en aparato crítico- algunas alternativas 
texto del Anonymus, que, como hemos dicho, contiene una paráfrasis del

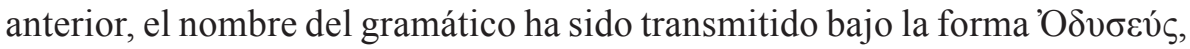

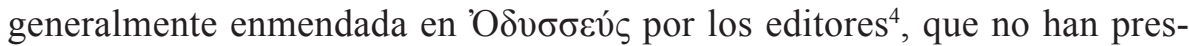
tado mayor atención a esa pequeña variación. Así las cosas, ésta constituye la única noticia que tenemos de la existencia de un gramático - metricólogo, según la cita - de nombre Odiseo, en la tradición literaria grecolatina. En las páginas que siguen trataremos de identificar a ese gramático, así como dar una explicación satisfactoria a su mención por parte de Longino.

El comentario de Longino, a juzgar por la parte conservada, debía de tener un alto contenido teórico, que fundamentaba y explicaba los conceptos, esquemas y clasificaciones métricas propuestos por Hefestión ${ }^{5}$. Además, no parece difícil perfilar los motivos que movieron a Longino a escribir su obra. Por un lado, el tratado de Hefestión, a pesar de ser, como es sabido, el más importante de este tipo escrito hasta ese momento, carece, sin embargo, de una base teórica que lo apoye y así, comienza in medias res, analizando inmediatamente la clasificación de los elementos que intervienen en la consecución de los distintos esquemas métricos ${ }^{6}$. Y en

al texto, en la idea de que podría tratarse de un error de transmisión, enmiendas que no son señaladas por ningún editor posterior, v. infra.

${ }^{4}$ El primer editor del tratado, Keil 1848, p. 12,6, mantiene la lectura original del manuscrito, mientras que ésta es corregida en las ediciones posteriores (v. Nauck 1867, p. 265, y Studemund 1886, p. 231, que ni siquiera menciona la variante en el aparato crítico).

${ }^{5}$ Cf. Brisson y Patillon 1998, pp. 3025-3028 ( Patillon y Brisson 2001, pp. 51-57).

${ }^{6}$ Sobre la organización del tratado, v. Ophuijsen 1987, pp. 11-13. La ausencia de fundamentación teórica en la obra de Hefestión parece tener una doble justificación. En primer lugar, el gramático bizantino del s. VI Jorge Querobosco (p. 180,11 ss. Consb.) señala en su comentario a la obra de Hefestión que éste había escrito primero 48 libros sobre los metros y que en tres etapas sucesivas los redujo a uno, que es el que ha llegado a nuestras manos (v. Pretagostini 1993, p. 373). La segunda razón está relacionada con el propio formato de la obra, un manual. Es muy probable que, como apunta Ophuijsen 1987, p. 12, el tratado estuviese diseñado como un libro de referencia para los propios estudiantes de Hefestión en Alejandría, como material de apoyo para las explicaciones del profesor. Ya Longino (fr. 42.100ss. P.-B.) señala que Hefestión no incluyó ningún tipo de explicación teórica sobre los elementos que iba a tratar de forma intencionada, pues la suya era una obra específicamente dirigida a estudiantes de métrica ya experimentados. Es más, según Longino (fr. 42.101 P.-B.), Hefestión reprochaba al metricólogo alejandrino Heliodoro, un siglo anterior, haber incluido en su obra, también sobre métrica — hoy perdida-, demasiadas explicaciones teóricas. 
segundo lugar, y quizás más importante, los estudios de métrica de Hefestión y otros académicos contemporáneos eran herederos de una larga tradición en la que existía una importante confusión teórica entre dos disciplinas, métrica rítmica y métrica filológica. En efecto, tras Aristóxeno, el primer teórico de la música en establecer una división clara entre música y poesía ${ }^{7}$, el estudio de la rítmica va a tomar caminos cada vez más divergentes, atendiendo bien a la música ( $\dot{v} \theta \mu$ iкoí) bien a la palabra escrita ( $\mu \varepsilon \tau \rho$ «оí). Esta división va a estar plenamente instaurada en época helenística en los grandes centros de estudio filológico. Cuando los estudiosos de las obras poéticas se ven obligados a atender a cuestiones relativas al verso, su organización y funcionamiento, se ven necesitados, en consecuencia, de una serie de principios metodológicos con arreglo a los cuales desarrollar su labor. Todo ello se lo suministraba la rítmica de los músicos. Sin embargo, al tratar de aplicar a la lengua escrita categorías y principios que surgieron para dar explicación a una realidad distinta, la música, el canto y la danza, se produjo un desajuste que hizo que los antiguos términos ahora aplicados a la métrica filológica fuesen tergiversados o despojados de su sentido originario ${ }^{8}$.

Longino, como decimos, es consciente de esta problemática que afecta a la métrica filológica (cf. fr. 42.131 P.-B.), y, especialmente, a la obra de Hefestión, que carece de fundamentos teóricos. Por ello, antes de abordar el comentario, considera necesario dedicar un apartado introductorio que deje establecida una clara distinción entre los dos conceptos, ritmo y metro. Es en este contexto cuando, tras definir el ritmo y señalar claramente su diferencia con el metro (fr. 42.1-56 P.-B.), menciona las distintas acepciones que habi-

\footnotetext{
${ }^{7}$ Por lo que se puede deducir de los testimonios anteriores a Aristóxeno, el ritmo aparece entonces estrechamente vinculado a la $\lambda \dot{\varepsilon} \xi 1 \zeta$, es decir, al componente lingüístico de aquella música vocal (sobre los teóricos de la música en época clásica anteriores a Aristóxeno, v. Wallace 1995). Fue Aristóxeno el primero en desligar esta implicación (cf. Aristox., Rhyth. 19 Pearson y Harm. p. 44,1ss. Da Rios) y en determinar que en términos musicales las sílabas, al no mantenerse siempre iguales a sí mismas, no pueden valer como unidades de medida, por lo que el sustrato del ritmo debía ser el propio tiempo, y así determina como unidad de

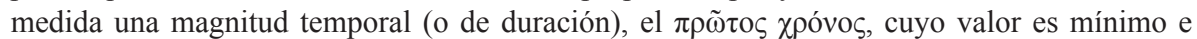
indivisible (cf. Aristox., Rhyth. 12 y 15 Pearson), v. Pearson 1990, p. xxxvi, Luque 1995, pp. 13-14, y Gentili y Lomiento 2003, p. 49.

${ }^{8}$ Gentili 1950, pp. 30-9, Koster $1966^{4}$, pp. 1-5, Luque Moreno 1995, pp. 11-12 у 59, у Gentilli y Lomiento 2003, pp. 17-9.
} 
tualmente tiene el término $\mu \varepsilon ́ \tau \rho o v$ en la teoría métrica filológica. En concreto señala cinco9:

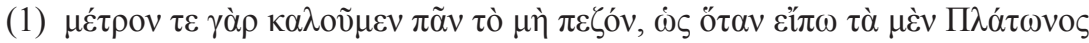

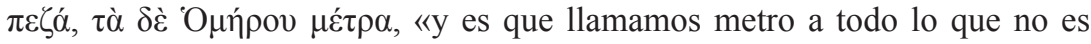
prosa, como cuando digo la prosa de Platón y la poesía (métra) de Homero»;

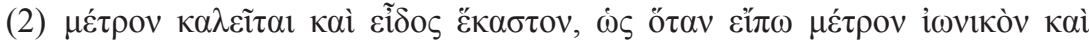

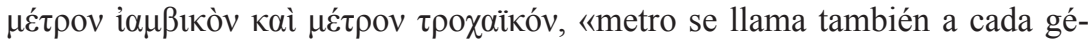
nero, como cuando digo metro jónico y metro yámbico y metro trocaico»;

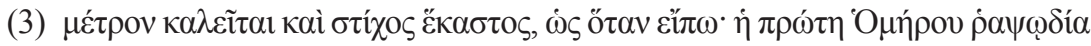

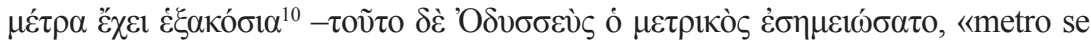
llama también a cada verso, como cuando digo el primer canto de Homero tiene 600 versos (métra) -sobre eso llamó la atención el metricólogo Odiseo»;

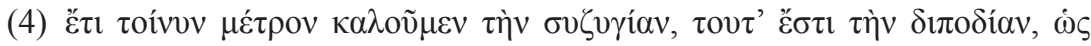

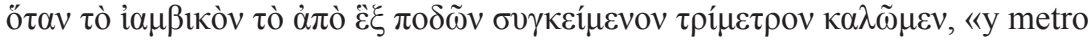
llamamos también a la sizigia, esto es, la dipodia ${ }^{11}$, como cuando llamamos trímetro al yambo compuesto de seis pies»;

${ }^{9}$ La edición de Consbruch 1906, p. 85,2-3 (cf. Patillon y Brisson 2001, p. 182,17 y

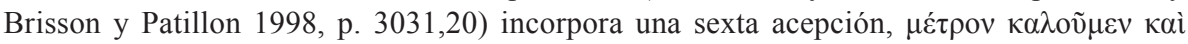

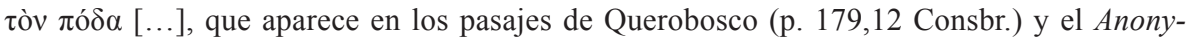
mus Ambrosianus de re metrica (p. 231,9 Studemund) que parafrasean a Longino, pero no en los manuscritos de éste. El añadido se incluye en la idea de que también debía de estar originalmente, pero se perdió en el curso de la transmisión del texto. Sin embargo, esa acepción, como muchas otras en la paráfrasis, debe de ser original de Querobosco, a quién también estaría siguiendo el Anonymus Ambrosianus, por lo que no parece necesario introducirla en el texto de Longino, cf. p. ej. Vaucher 1854, p. 298, Gaisford 1855², p. 146 y Westphal 1866, p. 87.

${ }^{10}$ Consbruch 1906, p. 84 (cf. Westphal 1866, p. 87, en aparato crítico) propone añadir tras $\dot{\varepsilon} \xi \alpha \kappa o ́ \sigma 1 \alpha<\kappa \alpha i ̀ ~ \delta \varepsilon ́ \kappa \alpha>$, siguiendo la paráfrasis del Anonymus Ambrosianus (p. 231,12

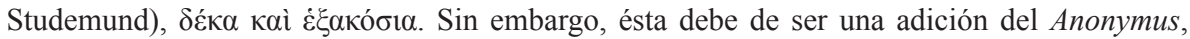
intentando aproximarse más al número de versos que contenía la versión de la Ilíada que conocía. Como señalan Patillon y Brisson 2001, p. 182, n. 2, es probable que Longino diera una cifra redonda y aproximada, teniendo en cuenta que en su época el número de versos de los cantos homéricos variaba de unas ediciones a otras.

${ }^{11}$ Se trata de una clara confusión entre dipodia y sizigia. La sizigia es un término técnico de la teoría rítmica musical que hace referencia a la combinación de dos pies simples y no

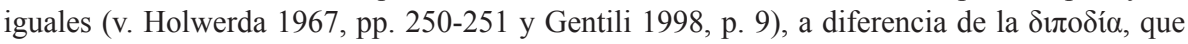
es la combinación de dos pies simples semejantes. Esta confusión fue muy común entre los metricólogos tanto griegos como latinos, v. Luque Moreno 1995, pp. 84-89. 


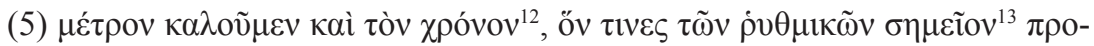

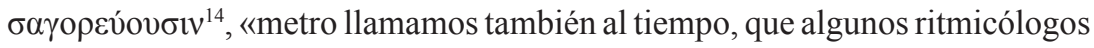
denominan sēmê̂on».

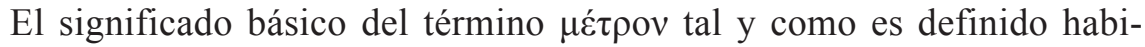
tualmente entre los teóricos griegos (cf. Aristid.Quint., p. 45,1ss. W.-I.; Heph. [v. Opuhijsen 1985, pp. 15-17 y 59-68]) hace referencia a una estructura particular del lenguaje compuesta por un sistema regular de sílabas breves y sílabas largas. De ahí se entiende que el término pueda haber llegado entre los metricólogos a aplicarse a cualquier tipo de estructura con una medida regular, independientemente de su tamaño. Ahora bien,

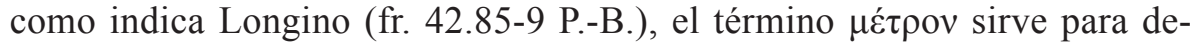

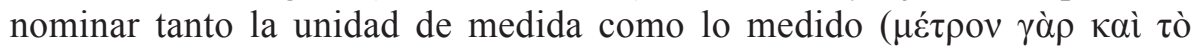

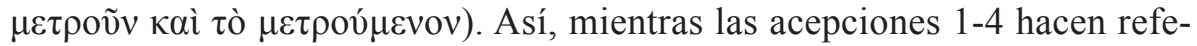
rencia a la estructura medida - poema, tipo de verso, línea, sizigia y dipodia, respectivamente-, la 5 hace referencia a la magnitud de medida, el tiempo. La mayor parte de estas acepciones son de uso habitual entre los metricólogos, incluso hay algunas que remontan a la fase rítmica arcaica

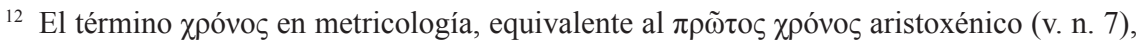
hace referencia a la unidad de medida mínima convencional, esto es breve = un tiempo, larga $=$ dos tiempos (v. Ophuijsen 1983, p. 55), de tal manera que, como señala a continuación, un hexámetro dactílico tiene 24 tiempos.

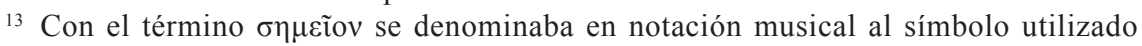
para señalar la unidad de tiempo más reducida, esto es, "“», el llamado $\pi \rho \tilde{\omega} \tau o \varsigma \chi \rho o ́ v o \varsigma$ (v. n. 7, cf. Pearson 1990, p. xxxviii, Michaelides 1978, pp. 238 ss.), pero también, a partir del uso descrito, pasa metonímicamente a ser usado como un mero sinónimo de

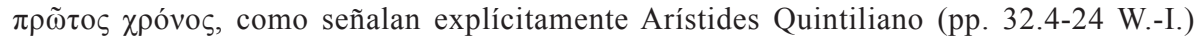
y Longino en este pasaje (cf. también Gentili y Lomiento 2003, p. 49). No obstante, parece que esta denominación fue utilizada no solo por los ritmicólogos, como indica Longino, sino también por algunos metricólogos, pues el propio Hefestión (pp. 12.15,

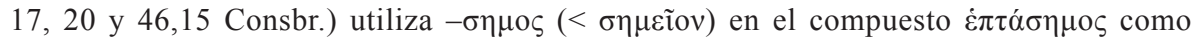

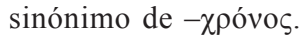

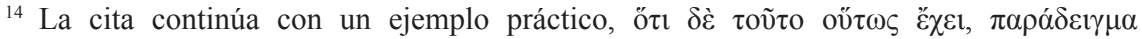

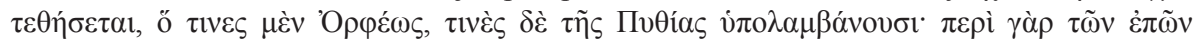

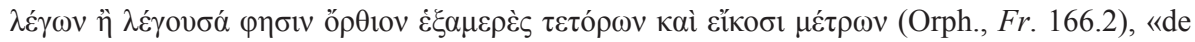
que eso es así, tenemos un ejemplo que algunos atribuyen a Orfeo y otros a la Pitia. Pues él o ella, hablando de versos épicos, dice: seis partes alineadas de veinticuatro medidas», que, además, no está exento de polémica; para una discusión sobre el origen de la cita, v. West 1983, pp. 231-23 y cf. Männlein-Robert 2001, p. 570. 
prearistoxénica ${ }^{15}$. Sin embargo, la número 3, la que establece $\sigma \tau i ́ \chi 0 \varsigma$ y $\mu \varepsilon ́ \tau \rho o v$ como sinónimos, precisamente la única a la que acompaña su autoría, es también la única que no está testimoniada en ninguna otra parte. Las fuentes antiguas son precisas al definir estos términos ${ }^{16}$; señalan de

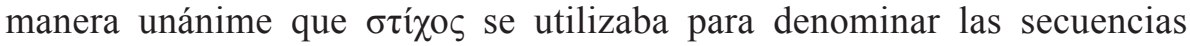
métricas superiores al dímetro, esto es, la unidad más pequeña dotada de autonomía propia, que, a su vez, recibía el nombre de $\kappa \tilde{\omega} \lambda \mathrm{ov}$. Las secuencias inferiores a éste último se denominaban кó $\mu \mu \alpha$. No obstante, fue el término $\kappa \tilde{\omega} \lambda$ ov el que acabó adquiriendo el significado genérico de «miembro», por lo que se usaba como sinónimo de otíxos. Así las cosas, todo

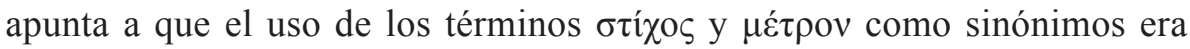
una novedad, con cierta singularidad además, ya que no se encuentra en ninguna otra fuente. Este hecho, sin duda, debió de ser uno de los motivos que llevó a Longino a mencionar al autor de la precisión terminológica,

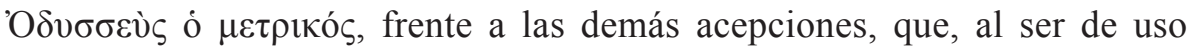
habitual, no necesitaban tal especificación. Sin embargo, la brevedad de la cita no permite saber qué razones llevaron a nuestro metricólogo a hacer

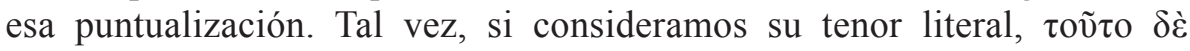

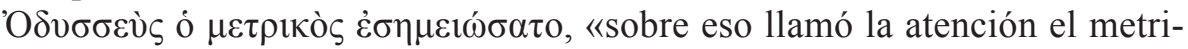
cólogo Odiseo», se limitara a constatar un hecho, la observación de un uso particular.

De los datos expuestos hasta el momento cabría deducir que el metricólogo Odiseo, obviamente anterior al s. III d.C., señaló un nuevo sentido del término $\mu$ ćt pov que no se encontraba en otros metricólogos ${ }^{17}$. Desde nuestro punto de vista, sin embargo, el hecho de que, por un lado, se desconozca la existencia de un gramático con ese nombre y que, por otro, «Odiseo» como antropónimo, fuera de la épica, sea prácticamente inexistente, es un indicio de que se trata de un error de transmisión textual.

Es algo irrefutable que existen numerosos autores de la Antigüedad cuya obra, incluso existencia, nos es desconocida por haberse perdido en el trans-

${ }^{15}$ Sobre la distinción entre prosa $(\pi \varepsilon \zeta \alpha ́$ ) y poesía $(\mu \varepsilon \dot{\tau} \rho \alpha)$, cf. Ar., $N u$. 638, Pl., Sph. 237a7, Grg. 502c.

${ }^{16}$ V. Heph., p. 63. Consbr., Schol. B ad Heph., p. 262.3 ss., Victorin.Gramm., GrL 6, p. 54,4 ss., Keil 1848, cf. Lomiento 1995, pp. 127-131, y Gentili y Lomiento 2003, p. 38, con bibliografía.

${ }_{17}$ Sic Brisson y Patillon 1998, p. 3031, n. 36 ( Patillon y Brisson 2001, p. 182, n. 3) y Männlein-Robert 2001, p. 569. 
curso de los avatares de la historia. Sin embargo, el hecho de que Longino cite expresamente a nuestro gramático en su comentario invita a pensar que se trataba de una figura relevante en el campo de la metricología, y que, por tanto, su existencia debiera estar registrada en algún otro lugar. Aparte de Hefestión, Longino tan sólo menciona específicamente otros tres autores

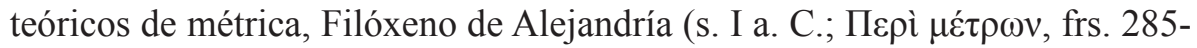

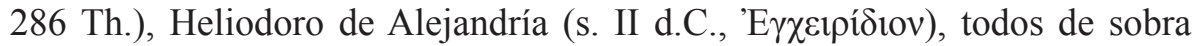
conocidos en la tradición, y al enigmático Odiseo. No vuelve a hacer referencia a ningún otro autor teórico individual, y ocasiones no le faltan en las que se limita a usar un vago colectivo ${ }^{18}$. La mención específica de estos gramáticos debe de responder al uso de sus obras por parte de Longino como fuentes para su tratado, o al menos para la parte introductoria, en lo relativo a la métrica filológica ${ }^{19}$. En este sentido, huelga decir de nuevo que no nos es conocida ninguna obra de ningún tipo de un metricólogo llamado Odiseo con el que poder contrastar la información dada por Longino.

Por otro lado, el rastreo sistemático de la antroponimia griega que ofrece el Lexicon of Greek Personal Names (LGPN) nos permite comprobar de hecho que, de entre los más de 35.000 nombres y apodos registrados hasta el momento en la documentación epigráfica y vascular en Grecia, el antropónimo Odiseo tan sólo está testimoniado en una ocasión en el sur de Italia ${ }^{20}$.

Creemos, por tanto, como venimos apuntando, que debe de tratarse de un error textual. Aunque los editores modernos del tratado de Longino y del

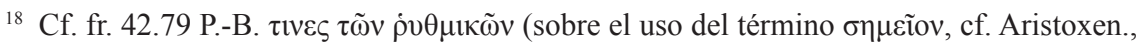
18 Ryth. Pearson, y v. Pearson 1990, p. xxxviii ad loc., Aristid.Quint., pp. 32.4-24 W.-I.); fr.

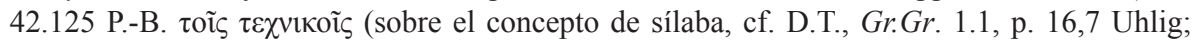

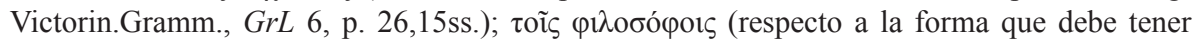
una definición correcta, cf. Arist., Top. 140a23 Ross).

19 Salvo para Hefestión, los datos que conservamos de las obras aludidas de estos autores es muy escaso, por lo que no es posible comprobar la forma en el que fueron utilizadas por parte de Longino; cf. infra sobre el supuesto metricólogo Odiseo.

${ }^{20}$ Además, el nombre está escrito en caracteres latinos. Otras variantes del nombre

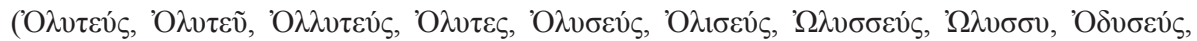

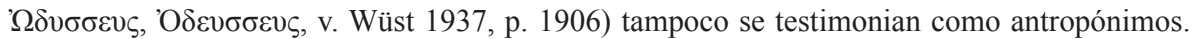
Únicamente dos formas derivadas, O $\Lambda \mathrm{Y} \Sigma \Sigma \mathrm{EI} \Delta \mathrm{A} \Sigma$ y $\mathrm{O} \Delta \mathrm{Y} \Sigma \Sigma \mathrm{EI} \Delta \mathrm{A} \Sigma$ cuentan con uno y dos testimonios, respectivamente. De hecho, la mayor parte de los nombres que aparecen en los poemas épicos rara vez son usados como antropónimos. Quizás esto sea debido a que se trata de nombres ficticios llenos de una carga semántica asociada al poema épico en el que aparecen, por lo que fuera de ahí no tuvieron fortuna (cf. Morpurgo Davies 2000, pp. 35-36). 
Anonymus Ambrosianus no hayan tenido en cuenta esta posibilidad, lo cierto es que ha habido otros filólogos que se mostraron partidarios de esta opción, si bien su contribución y comentario no aparece recogido en las ediciones posteriores. Así, ya J.A. Fabricius (ap. Gaisford $1855^{2}$, p. 146, n. c) señalaba que

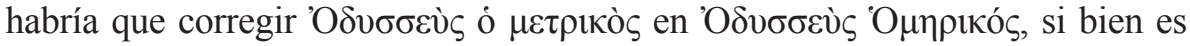
cierto que la corrección no aporta ninguna aclaración al sentido del pasaje.

Una contribución más significativa es la de Hotchkis (ap. Gaisford 18552, p. 146, n. c), autor de finales del s. XVIII, que llevó a cabo una edición comentada de Hefestión, por otro lado, inédita y que sólo nos es conocida a través de las notas de Gaisford en su edición (cf. 18552, pp. vii-viii). Hotchkis proponía tentativamente una ingeniosa aunque atrevida solución, basada en una supuesta imprecisión del texto de Longino, que omite el título de la obra concreta de Homero - Iliada u Odisea - a la que se está haciendo referencia

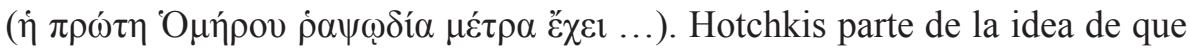

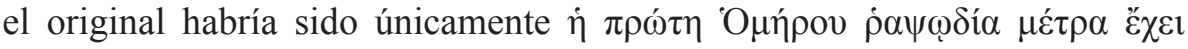
$\mathrm{YM} \Delta$, en referencia al primer canto de la Odisea, que tiene 444 versos. La

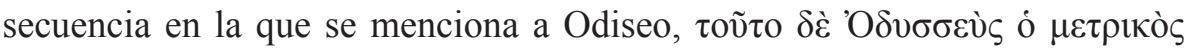
$\dot{\varepsilon} \sigma \eta \mu \varepsilon 1 \omega ́ \sigma \alpha \tau o$, habría sido en origen una glosa marginal, ahora muy corrupta, de un escoliasta posterior, que habría tratado de aclarar el sentido del texto

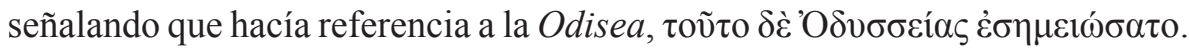
A partir de aquí, Hotchkis propone explicar la corrupción como una sucesión de errores. Según señala este autor, un copista posterior, al parecer muy poco dotado para su trabajo, habría integrado la glosa del escoliasta dentro del texto, al tiempo que habría malinterpretado tanto el original de Longino como la supuesta glosa. En primer lugar, el hecho de que no se especifique la obra concreta de Homero a la que se hace referencia habría dado pie a que el copista hiciese una lectura muy incorrecta del supuesto numeral original $<\mathrm{YM} \Delta>$. De este modo, habría interpretado como numeral sólo la primera letra $\langle\mathrm{Y}>$, que, además, entendió como $\langle\mathrm{X}>$, esto es, 600 , suponiendo así que esa nueva cifra hacía alusión al primer canto de la Iliada y no al de la Odisea. En cuanto a las otras dos letras del numeral $\langle\mathrm{M} \Delta\rangle$, que, de acuerdo con su peculiar razonamiento, no era posible interpretar como un número, el copista las habría considerado una abreviatura de ó $\mu \varepsilon \tau \rho ı$ cós. Finalmente, para aca-

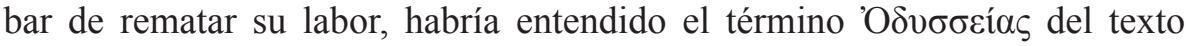

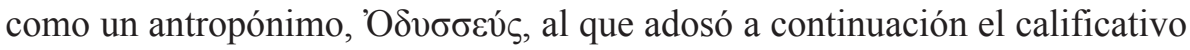

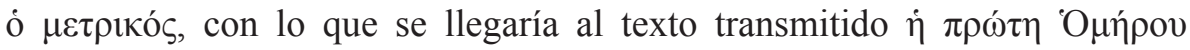

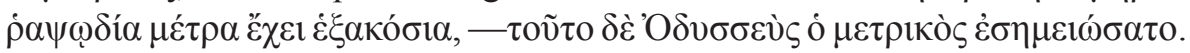


Como decimos, la hipótesis de Hotchkis carece de base, y no ha sido tenida en cuenta por ninguno de los editores modernos del texto, que, a excepción de Gaisford, ni siquiera la recogen en el aparato crítico. Y es que, aun

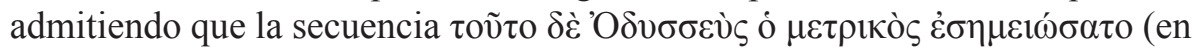

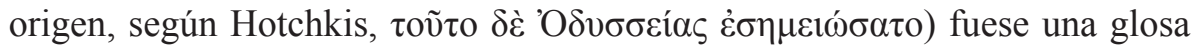
marginal, lo cual no parece para nada justificado ${ }^{21}$, aparte de la confusión de la primera letra del numeral, $\langle\mathrm{Y}\rangle$ por $\langle\mathrm{X}\rangle$, paleográficamente plausible, no se entiende (pace Hotchkis) de qué manera este copista por muy «minime peritus» que fuera, pudo haber llegado a causar tal confusión ${ }^{22}$ en un supuesto

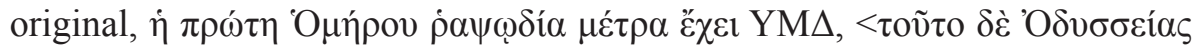
$\dot{\varepsilon} \sigma \eta \mu \varepsilon t \omega ́ \sigma \alpha \tau 0>$ (in margine).

Por las razones arriba indicadas, parece claro que en este contexto se mencionaba por su nombre propio a un autor teórico individual que había

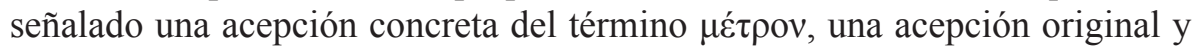
novedosa que, por tanto, no es posible atribuir a ningún autor conocido por nosotros, lo cual habría facilitado enormemente nuestra tarea de corrección. Ahora bien, en nuestra opinión, como venimos proponiendo, la forma que presenta en los manuscritos el nombre del metricólogo, Odiseo, parece deberse a un error de transmisión.

$\mathrm{Si}$, como defendemos, estamos ante un error de transmisión, éste debe de remontarse a una etapa antigua. En efecto, como señalábamos al principio,

${ }^{21}$ Cabe tener en cuenta que ésta constituiría la única glosa intercalada que se detecta en toda la obra de Longino. La hipótesis se basa, según el autor, en el hecho de que en los

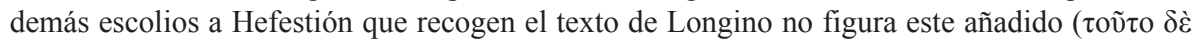

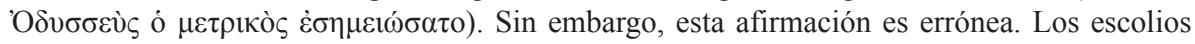
de la obra de Hefestión, tal y como fueron organizados por Westphal (1866, cf. también Hörschelmann 1881, pp. 262ss.), se dividen en dos grupos, denominados A y B. Pues bien, en los escolios A, aunque se hace alusión al pasaje concreto de Longino (cf. Sch. A ad Heph., p. 166,8-15), ésta no pasa de ser una somera referencia que sólo recoge su sentido general

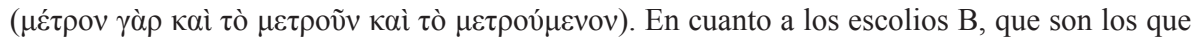
transmiten la parte conservada de la obra de Longino, en todos los manuscritos sí aparece la mención de Odiseo. Por otro lado, el comentario a la obra de Hefestión que hace Querobosco (pp. 175ss. Consbr.) se limita a parafrasear este pasaje Longino (p. 179 Consbr.) y no sólo no recoge la mención de Odiseo, sino ni siquiera la referencia homérica. En cualquier caso, Hotchkis no pudo conocer el Anonymus Ambrosianus de re metrica, publicado por primera vez en 1848 (H. Keil), que no sólo incluye estas palabras de Longino, sino que las parafrasea:

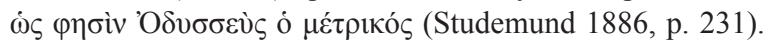

${ }^{22}$ Cf. Nauck 1865, p. 265, n. 18. 
este pasaje concreto de la obra de Longino se ha conservado en dos tradiciones manuscritas diferentes, una directa y otra indirecta. Por un lado, el grueso de la obra conservada - introducción y parte del inicio del comentario-, pertenece a una transmisión única, iniciada hasta donde podemos saber por el mejor y más completo códice conservado de la obra de Hefestión, el Ambrosianus I 8 (A), que está fechado entre los ss. XIV/XV. A continuación, el texto de Longino es transmitido también por las copias directas de aquél, $\mathrm{C}$ (Cantabrigiensis univ. Dd XI 70) y P (Parisinus 2881) ${ }^{23}$. La otra mención de este pasaje, aquella que hace el Anonymus Ambrosianus de re metrica, está contenida en un único códice, el conocido Ambrosiano C 222, y se remonta a una fecha más antigua, al s. XIII ${ }^{24}$. De acuerdo con esta reconstrucción, las dos tradiciones son copia, con cierta particularidad en cada caso, como veremos, de un arquetipo que ya contenía el error.

Teniendo en cuenta, pues, esa antigüedad a la que parece remontarse el error, el análisis paleográfico de la secuencia en la que se menciona a nuestro

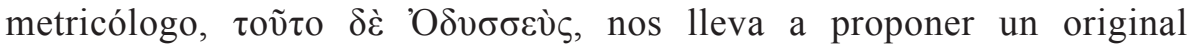
TOYTO $\Delta \mathrm{E} \Delta \mathrm{IONY} \Sigma \mathrm{IO} \Sigma$, en una fase en la que la escritura aún era mayúscula corrida. A partir de aquí, la corrupción habría sido originada por uno de los lapsus más habituales entre los copistas, la haplografía ${ }^{25}$. De este modo, en un momento dado de la transmisión del texto, en la secuencia original TOYTO $\Delta \mathrm{E} \Delta \mathrm{IONY} \Sigma \mathrm{IO} \Sigma$ se simplificó $\Delta \mathrm{E} \Delta \mathrm{I}$ en $\Delta \mathrm{E}$, escribiendo TOYTO $\triangle \mathrm{EONY} \Sigma \mathrm{IO} \Sigma$.

${ }^{23}$ Para una descripción del stemma de la obra y de la relación del tratado de Longino con los distintos escolios de Hefestión, v. Hörschelmann 1881, pp. 265-267, y Consbruch 1906, pp. vi-xxiv. Los datos indican que la obra de Longino pudo pervivir durante algún tiempo en la transmisión como obra individual, al menos hasta época bizantina medieval, cuando debió perderse. Los frs. 43-47 P.-B. de Longino, transmitidos por distintas fuentes bizantinas

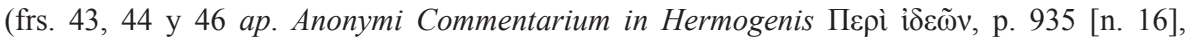
p. 982,15ss. y 984,4ss., respectivamente, Walz RhGr 7; fr. 45 ap. Hephaestionis Epitome, p. 360,18ss. Consbr.; y fr. 47 ap. Sch. A in Hepaestionem, p. 135,3 ss. Consbr.), contienen referencias al comentario de Longino a Hefestión (v. Patillon-Brisson 2001, p. 57, cf. íd. pp. 321-2, y Brisson y Patillon 1998, pp. 3034-3036; contra Männlein-Robert 2001, p. 362). En este sentido, es posible que también Querobosco (s. VI d.C.), cuando realizó su comentario a Hefestión (pp. 175ss. Consbr.), hubiese tenido acceso a la obra completa de Longino, no solo a la parte contenida en los escolios, pues éste es claramente la fuente de aquél en muchos aspectos (cf. Consbruch 1906 ad loc.).

${ }^{24}$ V. Nauck 1867, p. xxvi, y Studemund 1886, p. 212.

${ }^{25}$ V. p. ej. Bernabé $2010^{2}$, pp. 29-30. 
El siguiente paso posiblemente haya que situarlo en la copia del texto en escritura minúscula, con la consiguiente segmentación de las palabras. Así, el copista habría entendido que dicha secuencia se segmentaba como TOYTO$\Delta \mathrm{E}-\mathrm{ONY} \Sigma \mathrm{IO} \Sigma$, perdiéndose todo rastro del antiguo nombre y el posible origen de la corrupción. A continuación, el mismo copista u otro posterior, dado que $\mathrm{ONY} \Sigma \mathrm{IO} \Sigma$ es un antropónimo totalmente desconocido en Grecia,

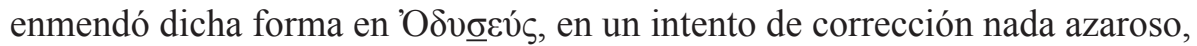
sino propiciado por la doble asociabilidad ${ }^{26}$ que sugiere el contexto; pues, por un lado, está la afinidad del contenido, y es que en las líneas anteriores se ha mencionado repetidas veces a Homero (11. 70 y 74) y, por otro lado, la asociación fonética, ya que el parecido entre los dos nombres es considerable.

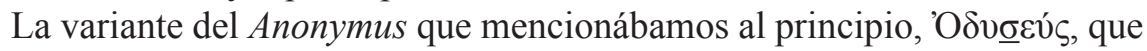
recordemos está registrada en una copia más antigua, es posible que esté reflejando un estadio intermedio de la confusión, $(\Delta \mathrm{I}) \mathrm{ONY} \Sigma \mathrm{IO} \Sigma$ y $\mathrm{O} \Delta \mathrm{Y} \Sigma \Sigma \mathrm{EY} \Sigma$, en el que la forma del nombre todavía no había adquirido su

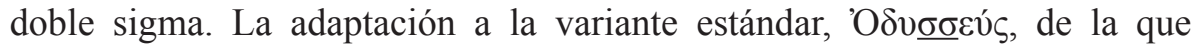

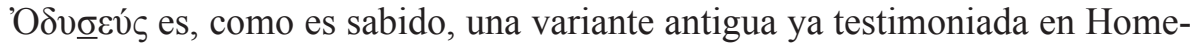
ro (v. n. 20), se habría producido bien por obra del copista del Ambrosianus I 8, bien en algún momento después de la separación de las dos tradiciones.

A partir de aquí, el hecho de que, como señalamos, la tradición manuscrita subsiguiente sea única explicaría que no haya vacilaciones en la cita, ya que los copistas no tenían ningún argumento para refutar dicha lectura, si acaso

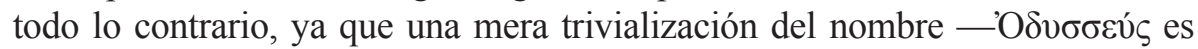
muy conocido en griego - debió de contribuir sin duda a perpetuar el error.

La enmienda hasta ahora propuesta, basada en argumentos puramente tex-

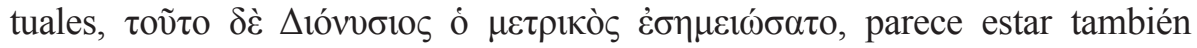
apoyada por los datos provenientes de la tradición gramatical griega, que ofrecen una identificación plausible para el autor, y contribuye, además, a aclarar la naturaleza de la cita en esta parte introductoria del tratado de Longino.

Partiendo, por las razones arriba apuntadas, del supuesto de que el gramático mencionado debió tener cierta fama en la Antigüedad - tanto como para ser mencionado por Longino como fuente, al nivel de Filóxeno y

26 Sobre los tipos y frecuencia de los errores provocados por el influjo de la asociabilidad, entendida como el conjunto de interferencias mentales que provocan en un copista la lectura del texto y su contexto, en la transmisión manuscrita, v. Timpanaro 1977, p. 101, y Sanz Morales 2011, p. 250. 
Heliodoro- , nos encontramos con que en la tradición gramatical griega hay cuatro candidatos con este nombre, que pudieron ser la fuente de Longino, esto es, anteriores al s. III d.C. y que trataron, o al menos pudieron tratar, cuestiones relacionadas con la métrica: Dionisio de Sidón ( $R E$ [135]), filólogo alejandrino de la segunda mitad del s. II a. C.; Dionisio de Fasélide $(R E$ [136]), filólogo, probablemente también alejandrino, de los ss. II-I a. C.; el rétor Dionisio de Halicarnaso, s. I a. C., y Dionisio, también de Halicarnaso ( $R E$ [142]), apodado el músico, del s. II d.C.

Entre las obras de Dionisio de Sidón y Dionisio de Fasélide se encuentran comentarios exegéticos a diversos poetas. No obstante, el hecho de que Lon-

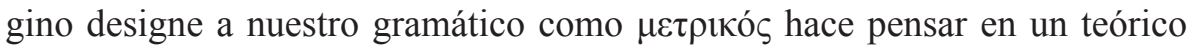
de la métrica, y no en un comentarista literario. Además, ninguno de los dos es identificado en las fuentes más que por su nombre, sin adición alguna en referencia a su ocupación. Por su parte, Dionisio de Halicarnaso, conocido teórico de la lengua, que trató asuntos directamente relacionados con la

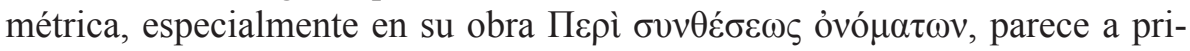
mera vista un buen candidato. Sin embargo, puede comprobarse de hecho en toda su producción literaria conservada que este autor siempre hace un uso escrupuloso de los términos $\mu \varepsilon ́ \tau \rho o v$ y $\sigma \tau i ́ x o \varsigma$, que nunca confunde y que distingue claramente en cuanto a su significado ${ }^{27}$. Si bien es posible que en una obra perdida hiciera referencia de algún modo a la precisión terminológica señalada por Longino, el hecho de que nunca aparezca mencionado como

${ }^{27}$ En contra de lo que sugiere Rhys Roberts 1910, p. 310, respecto a los distintos si-

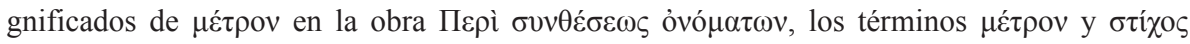
nunca se confunden. Los usos de $\mu \varepsilon ́ \tau$ cov en Dionisio de Halicarnaso se pueden agrupar en 4 acepciones: 1) referido a la poesía frente a la prosa (Comp. 3.1, 4.1, 4.14, 9.7, 10.2, 11.2, 19.2, 25.11; Dem. 36.3 [bis], 47.8, 48.3, 50.8); 2) referido a un esquema métrico en general o particular, como secuencia regular de sílabas largas y breves (Comp. 3.11, 4.2, 4.5, 17.11 19.3, 19.6, 19.7, 20.8, 20.21, 22.32, 25.7, 25.11 [bis], 25.16, 25.17, 25.21, 25.27, 26.2, 26.3, 26.4, 26.16; Dem. 50.6 [bis], 50.8 [bis], 50.9 [bis]; Isoc. 3.2; Lys. 3.7), que sólo o compuesto puede llegar a formar estructuras métricas complejas como los versos ( $\sigma \tau i ́ \chi 0 \varsigma)$, períodos ( $\pi \varepsilon \rho i ́ o \delta o \varsigma)$

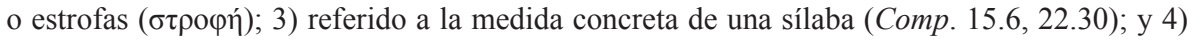
para distinguir el ritmo de la palabra escrita, $\mu \varepsilon ́ \tau \rho o v$, frente al ritmo musical, $\rho v \theta \mu o ́ \varsigma$ (Comp.

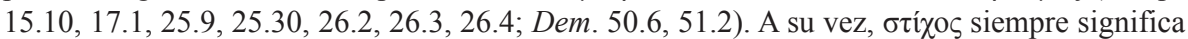

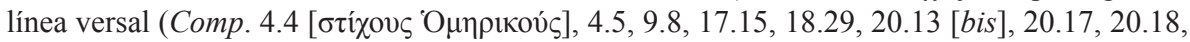
$20.21,25.16,26.2,26.4,26.11$ [bis] $26.12,26.13$ [quater]) compuesta por uno o varios $\mu \varepsilon ́ \tau \rho \alpha$ (19.3, 25.11 [bis]; Dem. 50.8 [bis]), e incluso en algunas ocasiones lo utiliza en referencia a una línea de prosa (Dem. 57.2; Th. 10.4 [bis], 19.1, 19.3, 33.1). 
metricólogo invita a barajar la cuarta posibilidad, Dionisio el músico, que, en efecto, tiene visos de ser la opción correcta.

Este otro Dionisio de Halicarnaso, músico teórico, trató ampliamente cuestiones directamente relacionadas con la rítmica. Conocemos algunas de

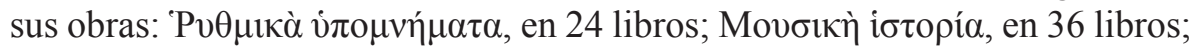

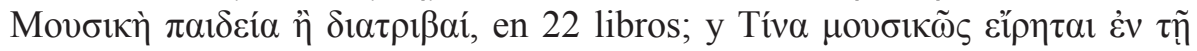

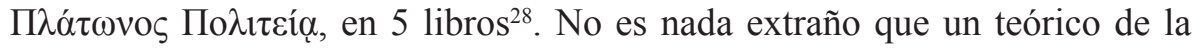

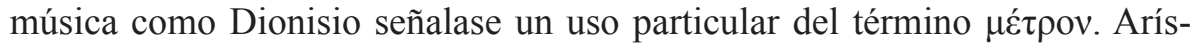
tides Quintiliano, por ejemplo, en su tratado De Musica, trata numerosas cuestiones de métrica filológica e incluso llega a definir el término $\mu \varepsilon ́ \tau \rho o v$ (p. 45 W.-I.), definición que es en todo similar a la concepción que se comprueba en Hefestión ${ }^{29}$.

Un análisis de la cronología y naturaleza de las fuentes de Longino parece aportar datos reveladores respecto a la identificación de nuestro gramático. Gracias al propio Longino (fr. 42.11, 12, 79 y 108 P.-B.) sabemos que para la redacción de su comentario usó como referencia los tratados de métrica de Filóxeno (s. I a. C.) y Heliodoro de Alejandría (s. I d.C.). Dado que las obras de ambos no se han conservado, no es posible conocer en detalle cuál es el grado de similitud y aceptación por parte de Longino de los presupuestos de aquéllos. No obstante, sabemos una vez más gracias al propio Longino (fr. 42.11-2, cf. 42.111-4 P.-B.) que Heliodoro en su obra llevó a cabo una completa definición de metro, definición que Hefestión, por las razones arriba indicadas, renunció a hacer, pero que, sin embargo, Longino considera del todo pertinente - de hecho la falta de contextualización en la obra de Hefestión es una de las motivaciones principales de su comentario-, en este caso para delimitar claramente su diferencia respecto al ritmo. En este sentido, es probable que ya Heliodoro abordase la enumeración de las distintas acepcio-

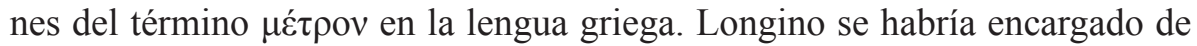
reelaborar esa enumeración, añadiendo la acepción correspondiente a Dionisio, obviamente no incluida en Heliodoro por ser este autor un siglo anterior a aquél. En este sentido se explica que Longino, al incluir una novedad respecto a su fuente, Heliodoro, señalase la autoría de la misma, siendo, además, precisamente esta acepción la única que cuenta con dicha especificación.

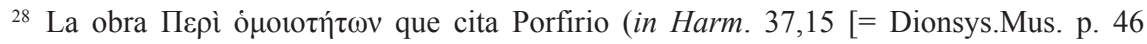

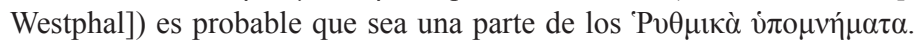

${ }^{29}$ V. Ophuijsen 1987, pp. 15-17 y 59-60. 
Por otro lado, el hecho de que aparezca mencionado, como defendemos, un teórico de la música como fue Dionisio, se aviene perfectamente con el contenido de esta introducción del comentario y arroja algo de luz sobre las fuentes y las relaciones intertextuales de la obra de Longino. Como ya dijimos en su momento, la rítmica postaristoxénica conlleva una clara división entre la música y la poesía, una división que provocó una importante confusión teórica entre los metricólogos. Longino, sin embargo, aunque situado en el lado de los estudios de la métrica, no pasa por alto la interrelación entre las ahora dos disciplinas y así lo hace constar en su introducción al comentario de la obra de Hefestión. En este sentido, si se tiene en cuenta, además, que, como dijimos, Longino tan sólo menciona específicamente a los autores teóricos que fueron sus fuentes, nos vemos inclinados a pensar que Dionisio podría haber sido la fuente de Longino en lo relativo al ritmo, del mismo modo que Hefestión, Filóxeno y Heliodoro lo fueron para la métrica.

Finalmente, restaría por explicar la presencia del calificativo ó $\mu \varepsilon \tau \rho \iota \kappa o ́ s ~$ que se añade al nombre de nuestro gramático. Dionisio, el autor que venimos proponiendo como corrección, sin embargo, nunca es mencionado de este modo en los textos, obviamente porque esa no era su ocupación. Ahora bien, resulta que Dionisio sí que contaba con un calificativo alusivo a su profesión,

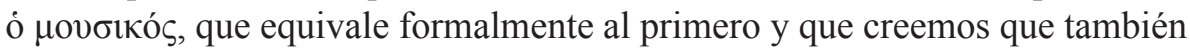
ha sido objeto de una corrupción. Este error, que aparece en las dos fuentes del pasaje, ya debía de estar, por tanto, en el arquetipo del que éstas derivan. El origen de la corrupción debe de ser bien un lapsus o bien una enmienda intencionada de un copista, que habría corregido $\mu \varepsilon \tau \rho$ iкós en lugar de

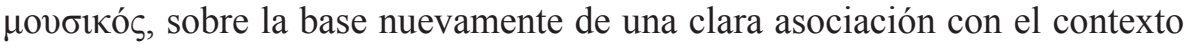

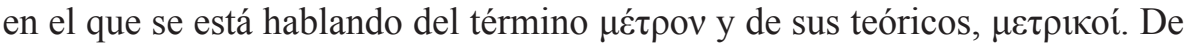

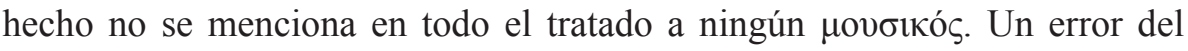
mismo tipo, aunque perteneciente a una transmisión textual más moderna, aquella asociada a la obra de Hefestión, aparece cuatro líneas más abajo (fr. 42.79 P.-B.). Aquí los manuscritos presentan una vacilación entre

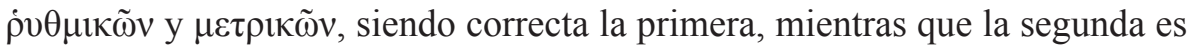
producto de una asociación contextual como la descrita más arriba.

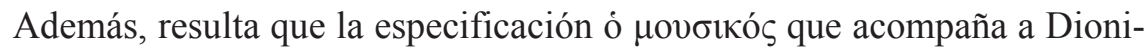
sio, que debió de surgir para individualizarlo frente a otros autores homónimos, aparte de en la Suda $\delta$ 1171, únicamente aparece en Porfirio (in Harm. 37.15 Düring), conocido alumno de Longino y claramente vinculado con él, 
por lo que es muy posible que este apelativo tuviera origen y fortuna en el ambiente académico de Longino.

Como hemos intentado demostrar, el comentario de Longino en este punto que estamos tratando parece presentar un problema textual, que ha sido obviado de manera sistemática por los distintos editores modernos, llevados de la mano por una transmisión manuscrita que era unánime y no presentaba variante alguna. En este caso es la singularidad del nombre del gramático la que nos ha hecho sospechar y considerar la corrección del texto transmitido. Creemos que la identificación propuesta para el enigmático y desconocido metricólogo Odiseo con el músico Dionisio es una solución paleográficamente plausible y convincente desde el punto de vista de la propia identificación. El hecho de que, por una lado, la confusión de su nombre se pueda explicar con argumentos puramente paleográficos bien tipificados, y que, por otro, los datos provenientes de su vida y obra vengan a coincidir de una manera coherente con la lectura propuesta y el contexto en el que se inserta, creemos que es un argumento lo suficientemente sólido como para apoyar la enmienda.

\section{BIBLIOGRAFÍA}

Bernabé, A. 2010²: Manual de crítica textual y edición de textos griegos, Madrid.

Brisson, L. y Patillon, M. 1998: «Longinus Platonicus Philosophus et Philologus II. Longinus Philologus», Aufstieg und Niedergang der römischen Welt, II 34.4, pp. 3023-3108.

Consbruch, M. 1906: Hephaesionis enchiridion, cum commentariis veteribus, Leipzig.

LGPN: Fraser, P. M. y Matthews E. (eds.) 1987-2013: A Lexicon of Greek Personal Names, vols. I-V, Oxford 1987-2013.

Gaisford, Th. 1855²: Hephaestionis Alexandrini Enchiridion, vol. 1, Oxford (1 $\left.{ }^{\mathrm{a}} \mathrm{ed} .1810\right)$. Gentili, B. 1950: Metrica greca arcaica, Mesina-Florencia.

Gentili, B. 1998: «Il commento di Eliodoro ad Aristofane, Ach. 285-336», QUCC 58, pp. 7-10.

Gentili, B. y Lomiento, L. 2003: Metrica e ritmica: storia delle forme poetiche nella grecia antica, Milán.

Holwerda, D. 1967: «De Heliodori comentario metrico in Aristophanem II», Mnemosyne 20, pp. 247-272.

Hörschelmann, W. 1881: «Untersuchungen zur Geschichte der griechischen Metriker. Die Composition der Hephestio-Scholien», RhM 36, pp. 260-301.

Keil, H. 1848: Analecta Grammatica, Halle. 
Koster, W. J. W. 1966": Traité de métrique grecque, Leiden.

Lomiento, L. 1995: «Il colon 'Quadrupede': Hephaest. Ench. p. 63, 1 Consbr., con alcune riflessioni sulla antica teoria metrica», QUCC 49, pp. 127-133.

Luque Moreno, J. 1995: De Pedibus, de Metris. Las unidades de medida en la rítmica y en la métrica antiguas, Granada.

Männlein-Robert, I. 2001: Longin, Philologe und Philosoph: eine Interpretation der erhaltenen Zeugnisse, Múnich.

Michaelides, S. 1978: The music of ancient Greece: an encyclopaedia, Londres.

Morpurgo Davies, A. 2000: "Greek Personal Names and Linguistic Continuity», en Hornblower, S. y Matthews, E. (eds.), Greek Personal Names: Their Value as Evidence, Oxford, pp. 15-39.

Nauck, A. 1867: Lexicon Vindobonense, San Petersburgo-Leipzig.

Ophuijsen, J. M. van 1987: Hephaestion on metre, Leiden.

Patillon, M. y Brisson, L. 2001: Longin, fragments. Art Rhétorique; Rufus, Art Rhétorique, París.

Pearson, L. 1990: Aristoxenus. Elementa Rhythmica, Oxford.

Pretagostini, R. 1993: «Le teorie metrico-ritmiche degli antichi. Metrica e ritmo musicale», en Cambiano, G., Canfora, L. y Lanza, D. (eds.), Lo spazio letterario della Grecia antica, vol. 1.2, Roma, pp. 369-391.

Rhys Roberts, W. 1910: Dionysius of Halicarnassus on literary composition, Londres.

Sanz Morales, M. 2001: «Tradición indirecta y error de memoria en crítica textual griega: observaciones metodológicas», AEF 34, pp. 237-252.

Studemund, G. y Shöll, R. 1886: Anecdota varia graeca et latina, vol. 1, Berlín.

Timpanaro, S. 1977: El lapsus freudiano: Psicoanálisis y crítica textual, Barcelona (trad. de la ed. ital., Il lapsus freudiano: Psicanalisi e critica textuale, Florencia, 1974).

Vaucher, L. 1854: Études critiques sur le Traité du Sublime et sur les écrits de Longin, París.

Wallace, R. W. 1995: «Music Theorists in Fourth-Century Athens», en Gentili, B. y Perusino, F. (eds.), Mousike. Metrica, ritmica e musica greca in memoria di G. Comoti, Pisa, pp. 17-39.

West, M.L. 1983: The Orphic Poems, Oxford.

Westphal, R. 1866: Scriptores metrici graeci, vol. 1, Leipzig.

Wüst, E. 1937: «Odysseus», RE 17.2, cols. 1905-1996.

Fecha de recepción de la primera version del artículo: 30/07/2015

Fecha de aceptación: 18/09/2015

Fecha de recepción de la version definitiva: 29/09/2015 